\title{
DIRECT GEOREFERENCING OF UAV DATA BASED ON SIMPLE BUILDING STRUCTURES
}

\author{
W. Tampubolon*, W. Reinhardt \\ AGIS, Institute for Applied Computer Science, Universität der Bundeswehr München, Germany - (winhard.tampubolon, \\ wolfgang.reinhardt)@unibw.de
}

Commission I, ICWG I/Vb

KEY WORDS: image orientation, structure from motion, tie points, building structures

\begin{abstract}
:
Unmanned Aerial Vehicle (UAV) data acquisition is more flexible compared with the more complex traditional airborne data acquisition. This advantage puts UAV platforms in a position as an alternative acquisition method in many applications including Large Scale Topographical Mapping (LSTM). LSTM, i.e. larger or equal than 1:10.000 map scale, is one of a number of prominent priority tasks to be solved in an accelerated way especially in third world developing countries such as Indonesia.

As one component of fundamental geospatial data sets, large scale topographical maps are mandatory in order to enable detailed spatial planning. However, the accuracy of the products derived from the UAV data are normally not sufficient for LSTM as it needs robust georeferencing, which requires additional costly efforts such as the incorporation of sophisticated GPS Inertial Navigation System (INS) or Inertial Measurement Unit (IMU) on the platform and/or Ground Control Point (GCP) data on the ground. To reduce the costs and the weight on the UAV alternative solutions have to be found.

This paper outlines a direct georeferencing method of UAV data by providing image orientation parameters derived from simple building structures and presents results of an investigation on the achievable results in a LSTM application. In this case, the image orientation determination has been performed through sequential images without any input from INS/IMU equipment. The simple building structures play a significant role in such a way that geometrical characteristics have been considered. Some instances are the orthogonality of the building's wall/rooftop and the local knowledge of the building orientation in the field. In addition, we want to include the Structure from Motion (SfM) approach in order to reduce the number of required GCPs especially for the absolute orientation purpose.

The SfM technique applied to the UAV data and simple building structures additionally presents an effective tool for the LSTM application at low cost. Our results show that image orientation calculations from building structure essentially improve the accuracy of direct georeferencing procedure adjusted also by the GCPs. To gain three dimensional (3D) point clouds in local coordinate system, an extraction procedure has been performed by using Agisoft Photo Scan. Subsequently, a Digital Surface Model (DSM) generated from the acquired data is the main output for LSTM that has to be assessed using standard field and conventional mapping workflows. For an appraisal, our DSM is compared directly with a similar DSM obtained by conventional airborne data acquisition using Leica RCD-30 metric camera as well as Trimble Phase One (P65+) camera. The comparison reveals that our approach can achieve meter level accuracy both in planimetric and vertical dimensions.
\end{abstract}

\section{INTRODUCTION}

The role of geospatial data as an important basis of government think-tank has been profoundly developed since 2011 by the legislation about Geospatial Information Act in Indonesia. This ambitious act urges the availability of large scale topographical maps up to the 1:1,000 map scale level. Large scale topographical maps are frequently associated with high resolution spatial data. This type of data can support the national development e.g. related to disaster preparedness, detail spatial planning, etc. Unfortunately, in order to provide high resolution 3D geospatial data, large scale topographical mapping still relies on conventional airborne campaigns, which is in general a costly but not timely mapping project. The acquisition of topographic data has been nowadays not only limited to the conventional methodologies such as terrestrial survey, aerial photogrammetry and remote sensing technologies. In specific, Indonesia as one of the archipelagic countries around a disaster prone area requires topographic data and corresponding processing techniques as a framework for supporting disaster preparedness and quick emergency response. Geospatial data are mandatory in this case because they contain fundamental geospatial features especially of the earth surface (also called terrain) information with respect to its proper geometrical accuracies. During disaster and emergency situations, geospatial data can provide important information for decision support systems. As one instance of basic geospatial data, large scale topographic maps are essential in order to enable accurate analysis within quite a number of societal challenges. Recently, the utilization of geospatial data using topographic maps as a basic reference is mandatory to support accurate quick regional mapping in a scale of 1:5,000 in so called "village mapping". The combination between accuracy requirements and time restriction is considered as critical in this activity. Therefore, this paper outlines a direct georeferencing method of UAV data by providing image orientation parameters derived from simple building structures and presents results of an investigation on the achievable results in a LSTM application. In this case, the image orientation

* Corresponding author 
determination has been performed through sequential images without any input from INS/IMU equipment.

\subsection{Structure from Motion (SfM) using UAV}

Structure from Motion is the prominent approach to provide the full three-dimensional structure of an object viewed from a wide range of positions by taking into account the sensor movement along its trajectory (Westoby, 2012). This approach can provide the camera position as well as the object geometries without any knowledge as required in the conventional softcopy photogrammetric methods such as the 3D position of the camera or a set of Ground Control Points (GCP) or camera calibration in the worst case.

Since the last decade, UAV technologies have showed a tremendous increase in the application, providing a basis to develop new alternative platforms for analyzing and monitoring earth surfaces. Among the available technologies, UAV platform has a significantly rising number of applications and innovations.

$\mathrm{UAV}$ as an alternative platform for geospatial data acquisition offers potentials because of its flexibility and practicability combined with low cost implementations. After all, the high resolution data collected from UAV platforms have the capabilities to provide a quick overview of one region. Nevertheless, there are some limitations that shall be taken into account in the UAV data processing for topographic mapping.

In this paper, we present an approach of SfM of UAV based data for topographical mapping which uses building structures to determine the orientation parameters. The role of GCPs is reduced in our approach since its availability and accuracy are mostly problematic in the Indonesian region.

\subsection{Research objectives and motivation}

The main advantage of UAV platforms is the capability to enable on-demand very high resolution data collection which can be customized efficiently. Frequently, UAVs use nonmetric camera sensor aiming for point cloud data extraction as well as orthophoto generation.

In general, direct georeferencing requires sensor orientation parameters measured from on-board sensors in order to be able to transform image coordinates to absolute ground coordinates without any field GCP. Unfortunately, because of the payload limitation and cost constraints, it is generally difficult to include a complete IMU and INS system with high-end accuracy on the UAV platform.

Geospatial data collected from a UAV are usually captured from low altitude. Under this circumstance, there normally is a significant occlusion surrounding the elevated objects such as high buildings, skyscrapers, towers, etc. This situation usually brings a tedious work in the data processing, especially during mosaicking tasks. However, these elevated objects can be useful if we use their 3D shapes and forms as a reference model to precisely extract image orientation parameters.

As already presented by Zhang, 2004, the elevated objects such as buildings and other man-made objects can be used to extract precise camera parameters for sequential images. At this point, we are going further to use these camera parameters as an input to adjust sensor orientation from the GPS and IMU/INS equipment.

In this paper, we want to demonstrate that a UAV platform can deliver geospatial data with sufficient accuracy to be used for LSTM by only using a minimum amount of GCPs. For this purpose, a direct georeferencing method has been selected as a potential solution to overcome the dependency from GCPs.

In our approach, we use only a GPS tag from the camera combined with minimum amount of GCPs. For our use case, we want to investigate the role of building structures incorporated into the georeferencing method. Our main motivation is to use camera orientation parameters derived from SfM algorithm in the conventional aerial triangulation based on building structures for providing the external orientation parameters.

Firstly, this research focuses on the comparison of different georeferencing method for photogrammetric data. In order to evaluate the results, it is necessary to compare available data with various geometric accuracies. We investigated not only UAV data but also airborne data acquisition equipped with digital medium-format photogrammetric camera as well as sensor position/orientation measurement unit (Figure 1). At this end of this part, the reference data has been selected based on the Independent Check Point (ICP) Level 1 result. The objective of ICP Level 1 is to assess the geometric accuracy for each data referring to the GNSS measurements.

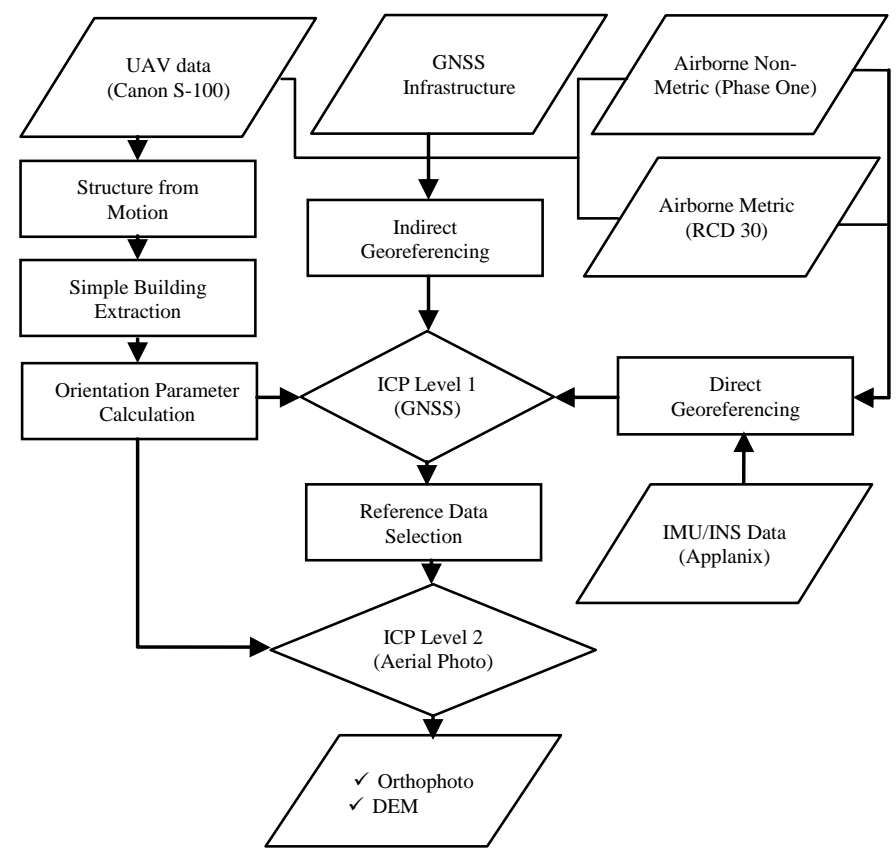

Figure 1. Workflow applied for this paper

Secondly, another objective of our investigations is to define a proper method for the purpose of image orientation extraction from high resolution UAV data. A SfM approach also considering building structures has been chosen as an alternative way to detect sensor orientation with high accuracy. On ICP Level 2, the results from each approach is subsequently validated against reference data acquired from selected reference data either from airborne campaign using Leica RCD30 (metric camera) or Trimble Phase One (P65+). 
Finally, this research also presents the achievable direct georeferencing results for different sensors / platforms.

\subsection{Area of Interest}

Our test area around the Geospatial Information Agency of Indonesia (BIG) office has been selected because of the availability of reference data, including the geodetic reference network infrastructure.

In general, the test site covered approximately a building area of 5 hectares which has an approximate elevation of 140 meters above mean sea level (msl). The terrain condition of the study area such as building, trees, roads, etc. is classified as flat with a lot of building structures (see Figure 2).

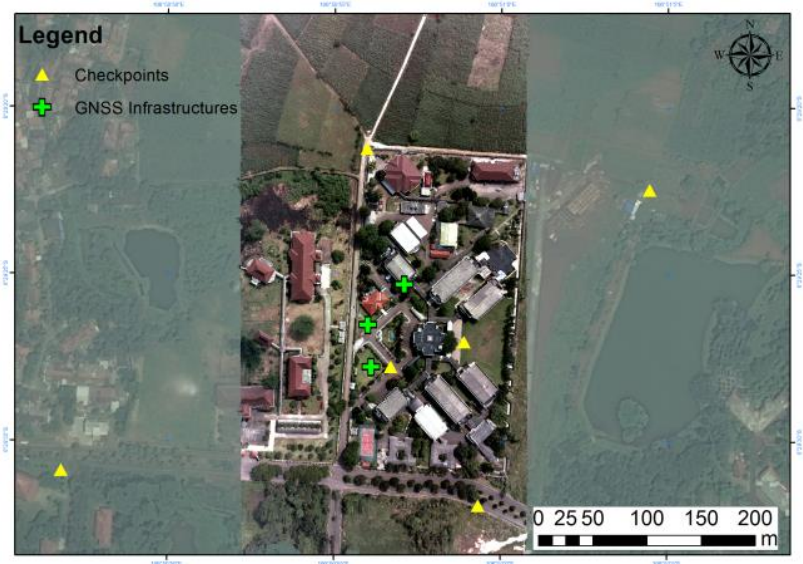

Figure 2. Area of Interest (BIG's office, Indonesia)

\section{RESEARCH METHODOLOGY}

Direct georeferencing is defined as a method which provides the orientation parameters of the camera only through on-board sensors (e.g. GPS, IMUs) without any field GCP. This term is traditionally used in the context of processing aerial photogrammetric data (Cramer, 2000) but recently also in conjunction with UAVs.

\subsection{Data Acquisition}

Until recently, UAV technology is still improving its performance in order to comply with conventional geospatial data requirements and specifications. Instrumentation control, navigation and sensors are the most prominent aspects to be taken into account.

With respect to its carrying capacity, usually UAV platforms use only consumer's grade camera as a main sensor especially for mapping purposes. One day data acquisition from approximately $268 \mathrm{~m}$ above ground level (agl) has been performed using Skywalker 1680 with wingspan $1,720 \mathrm{~mm}$ to photograph the test area.

This relatively high altitude has been selected in order to minimize the required time for the data acquisition as well as the subsequent data processing. However, the resolution of UAV data is still compatible with the aerial photogrammetric method as further discussed in section 3.1.
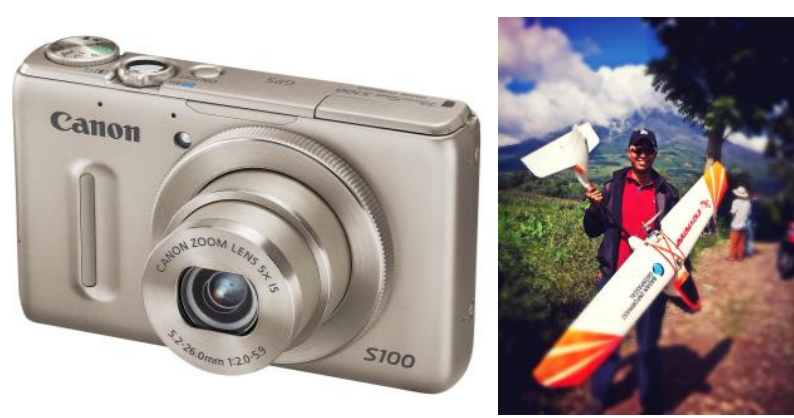

Figure 3. UAV fixed wing and camera used (left: Skywalker 1680, right: Canon S-100)

The above mentioned fuselage (Figure 3 ) is capable enough to carry the digital cameras used in this project i.e. Canon S-100 (Table 1) including the necessary power sources. This acquisition captured approximately 64 selected full-color aerial (optical) frames with more than $85 \%$ overlap and $45 \%$ sidelap which can be sufficiently processed by using the photogrammetric approach.

\begin{tabular}{|l|c|}
\hline Camera & Canon S-100 \\
\hline Weight & $198 \mathrm{~g}$ \\
Resolution & 12 Mpixels \\
Focal length & $5.2-26 \mathrm{~mm}$ \\
Optical zoom & 5 times \\
Sensor size & $7.44 \times 5.58 \mathrm{~mm}$ \\
Image size & $\pm 3.5 \mathrm{Mb}$ \\
\hline
\end{tabular}

Table 1. Camera specification

Höchle, 2012 has concluded that for example Leica RCD30 camera can be used for the generation of city models and automated Digital Surface Model (DSM) with a comparable quality to the large-format photogrammetric camera. From this point of view, the use of a medium-format camera for our goals is adequate enough.

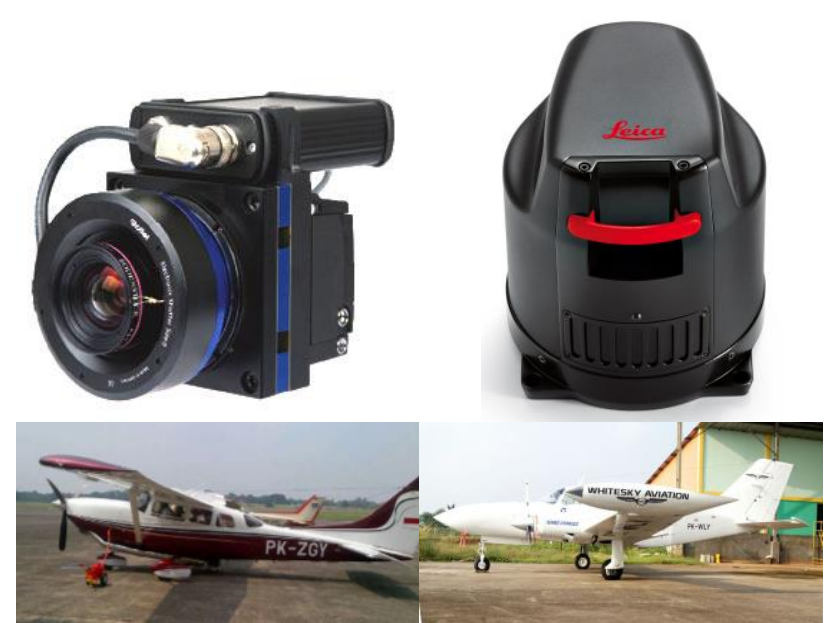

Figure 4. Aircraft and cameras used (left : Cessna 206t with Trimble P65+, right : Cessna 402b with Leica RCD30)

Therefore, airborne campaigns using each Leica RCD30 and Trimble Phase One (P65+) camera has been performed in May 2013 and August 2011 to provide aerial photogrammetric data (Figure 4). This acquisition used Cessna aircraft fully equipped by Gyro-stabilizer as well as the IMU/INS to support accurate direct georeferencing. Table 2 includes the details of the three flight missions. 


\begin{tabular}{|c|c|c|c|}
\hline & $\begin{array}{l}\text { UAV Canon } \\
\text { S100 }\end{array}$ & Leica RCD30 & $\begin{array}{l}\text { Trimble Phase } \\
\text { One (P65+) }\end{array}$ \\
\hline Altitude & $268 \mathrm{~m}$ agl. & $500 \mathrm{~m}$ agl. & $793 \mathrm{~m}$ agl. \\
\hline Aircraft & Skywalker 1680 & Cessna 402b & Cessna 206t \\
\hline Focus & $5.712 \mathrm{~mm}$ & $53 \mathrm{~mm}$ & $51.407 \mathrm{~mm}$ \\
\hline Date & 12 June 2014 & 9 May 2013 & 22 August 2011 \\
\hline $\begin{array}{l}\text { On-board } \\
\text { Navigation }\end{array}$ & GPS Camera & $\begin{array}{l}\text { Leica IPAS20 } \\
\text { (IMU/INS) }\end{array}$ & $\begin{array}{l}\text { Applanix POS } \\
\text { AV (IMU/INS) }\end{array}$ \\
\hline $\begin{array}{l}\text { GPS/IMU } \\
\text { Accuracy }\end{array}$ & $\begin{array}{c}\text { Position: } 10-20 \\
\text { m }\end{array}$ & $\begin{array}{c}\text { Position: } 0.05- \\
0,3 \mathrm{~m} \\
\text { Roll\&Pitch: } \\
0.008^{\circ} \\
\text { Heading: } 0.015^{\circ}\end{array}$ & $\begin{array}{c}\text { Position: } 0.5-2 \\
\text { m } \\
\text { Roll\&Pitch: } \\
0.03^{\circ} \\
\text { Heading: } 0.28^{\circ}\end{array}$ \\
\hline
\end{tabular}

Table 2. Acquisition details

By using this type of aircraft, the altitude during acquisition can be set up to $500 \mathrm{~m}$ agl to acquire photogrammetric data with the best possible resolution. Therefore it is sufficient to use this photogrammetric data as a source to derive the building structures mentioned by digitizing them.

\subsection{Georeferencing method}

There are two types of georeferencing applied for our project:

- For indirect georeferencing of all captured data, we use the geodetic and geodynamic control of certain geospatial references system i.e. Indonesian Geospatial Reference System (SRGI).

- For direct georeferencing of the airborne data, we use a GPS IMU/INS on board.

Based on the previous investigation presented in (Tampubolon, 2014), the minimum amount of GCPs to deliver sub-meter level of accuracy are 6 for each single image. From this context, we want to reduce the number of GCPs and only used three GNSS monitoring stations (BAKO, BAK1, BAK2) as the GCPs instead. As depicted in Figure 2. Area of Interest (BIG's office, Indonesia), the distribution of the GCPs is also centralized in the center of the test area because we want to reduce the GCP dependency in our approach as well.

The main reason to use GNSS monitoring station (Figure 5), as the GCPs in this project is to ensure the $3 \mathrm{D}$ accuracy. Since the three aforementioned GNSS-points are also dedicated for the Continuous Operating Reference System for the global monitoring service, the positional accuracy is in the range of millimeter level. Their $2 \mathrm{D}$ positions as well as their height (above ellipsoid/msl using precise geoid model) can also be accessed freely via internet at http://www.srgi.big.go.id.

In addition, to confirm the results we use the direct georeferencing data for each of the two airborne missions described above. For the purpose of independent validation of horizontal accuracy, additional 10 Independent Check Points (ICP) were conducted using GNSS in sub-centimeter accuracy.

To determine any significant accuracy of elevation improvement provided by the use of building structure objects, the georeferencing was performed by using two image processing software. Agisoft Photo Scan was used to generate the Ortophoto and DEM by indirect georeferencing method while PCI Geomatics was used to perform orientation parameter extraction using building structures.
Finally, the generated DEMs have been evaluated against the ICP data as further explained in section 3.1. At the end, the best available existing DEM i.e. Leica RCD30 DEM will be used as the reference data for accuracy assessment of the UAV data.

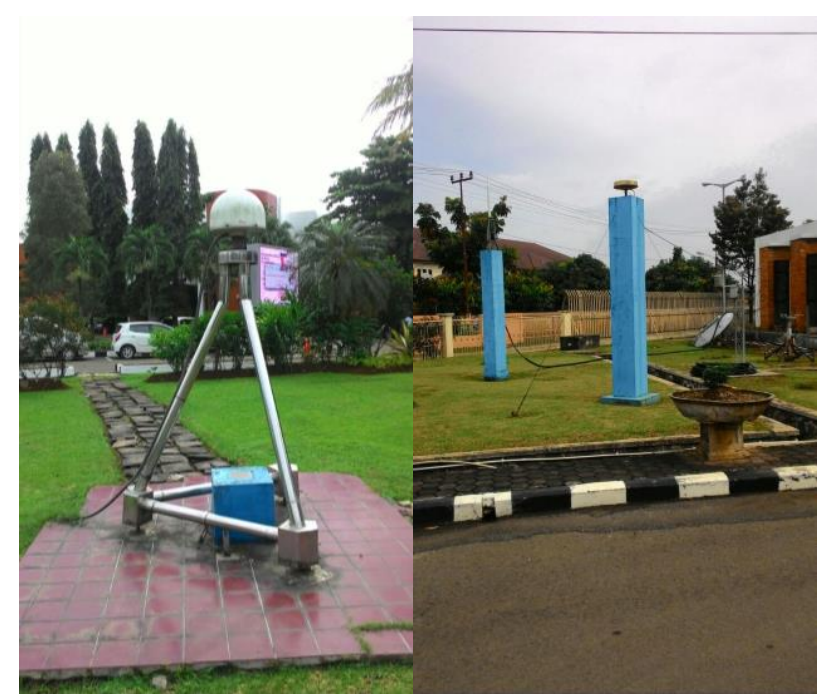

Figure 5. Geodetic Control (CORS) in BIG's office

\subsection{Simple Building Structures}

The simple building structures play a significant role in such a way that local geometrical knowledge can help to add more conditions in the computational equations. Some instances are the orthogonality of the building's wall/rooftop and the local knowledge of the building orientation in the field.

The previous SfM applied result using Agisoft Photo Scan from (Javernick, 2014) indicates georeferencing errors in the level of decimetre $(0.04 \mathrm{~m})$ for planimetric and centimetre $(0.10 \mathrm{~m})$ for elevation in a non-vegetation areas. This result endorses us to improve the orientation parameters from Agisoft Photo Scan by taking into account building structures in the subsequent bundle adjustment using PCI Geomatics.

In our approach, the mosque has been selected as a building reference object for orientation parameter determination in the next section. In addition to the orthogonality of the rooftop, the main reason of this selection is that the mosque has its regular building direction on the ground (Figure 6). This regularity makes an exclusive input to our algorithm without any necessary measurement on the field.

The building outlines are manually digitized using 3D Summit Evolution stereoplotter in $\mathrm{mm}$ height resolution to be used for the orientation parameters improvement. The absolute accuracy of these data, captured from the Leica RCD30 imagery, is $1.55^{*} \mathrm{GSD}=7.75 \mathrm{~cm}$. As an example for the mosque, we captured 8 edge points of the rooftop with additional 4 orthogonal lines and 4 edge lines (Figure 6). 


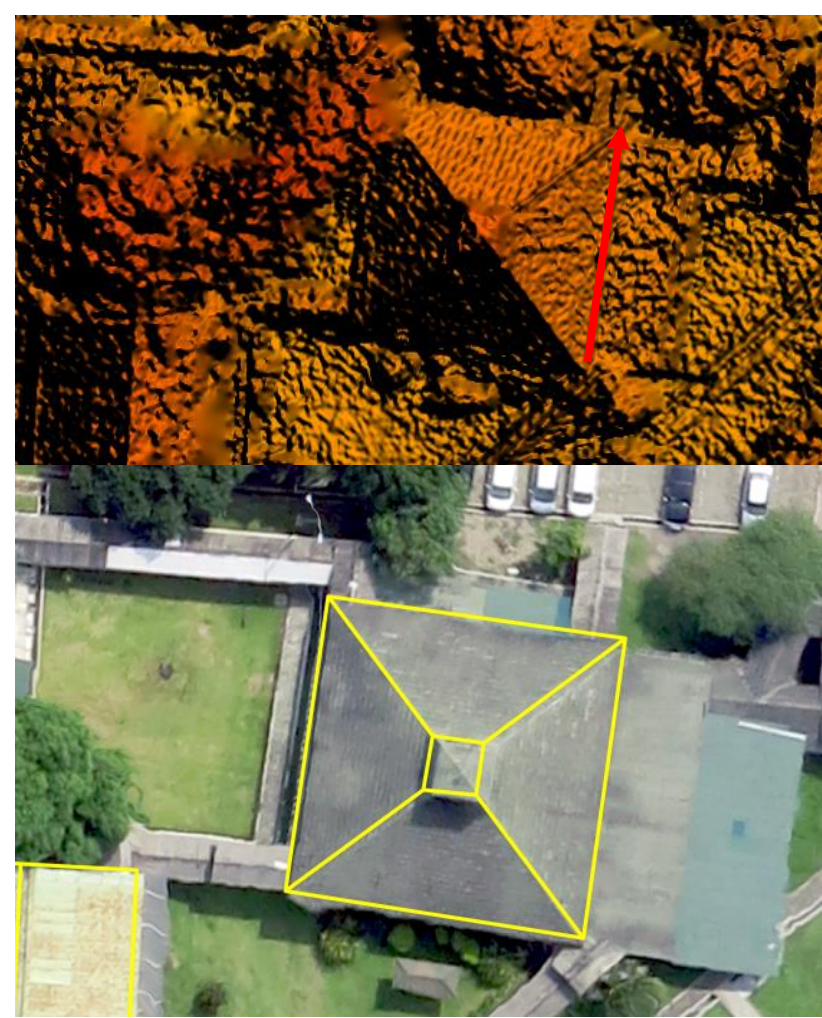

Figure 6. Mosque building object : DEM (upper), building outlines (lower)

Agisoft Photo Scan is a photo processing software that can be used to apply SfM approach. This software implements robust feature matching algorithm across the images. Firstly, Agisoft Photo Scan detects points in the source images which are relatively stable from different viewing points as well as lighting sources including their descriptors. Finally, these descriptors are used to reconstruct the structures across the sequential images (Agisoft, 2013).

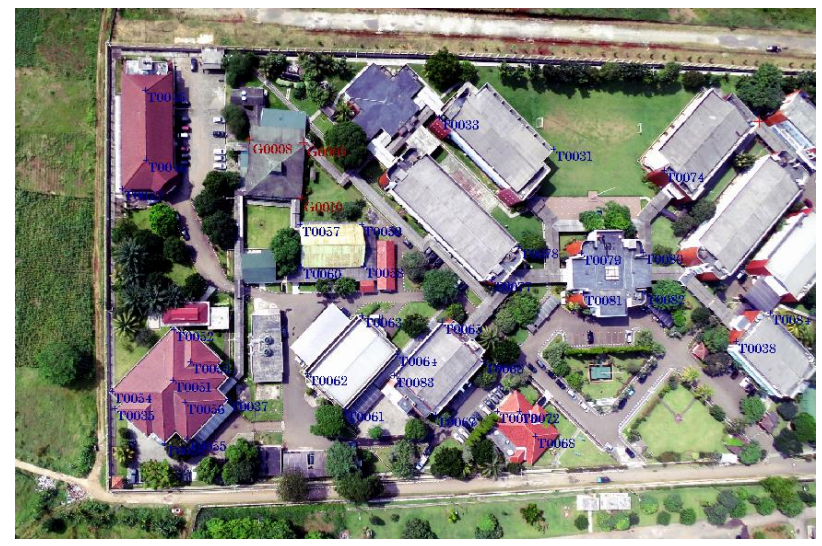

Figure 7. Simple Building Structure Consideration

The first step to derive building model from our UAV data is the internal orientation calculation by using Agisoft Photo Scan within our sequential images. The GPS tag camera as read from Exchangeable Image File Format (EXIF) metadata is used as an approximate principal point to perform initial bundle adjustment for self-calibration of camera parameters. Hence, the 3D model for each building structure in a rough absolute coordinates has been extracted from some pairs of images by using Agisoft Photo Scan.
In the next step, we consider a mosque in a simple building form and direction in order to estimate the elevation of the other buildings in our test area by taking into account the relief displacements. This height estimation is used to improve the external orientation camera of each image. For this purpose we use PCI Geomatics in order to manually extract the set of Tie Points on each edge of the building structures (Figure 7). Hence, this manual process focuses only on building structures instead of other objects.

\subsection{Point Cloud extraction}

Basically our main objective is to extract relevant image orientation parameters for supporting direct georeferencing method of UAV data. The SfM technique applied to simple building structures presents an effective tool for the LSTM application at low cost. Initial results show that image orientation calculations from building structure essentially improve the accuracy of direct georeferencing procedure provided from GCPs data. The extraction of three dimensional (3D) point clouds in a local coordinate system has been performed by using Agisoft Photo Scan.

In this paper, there are two approaches introduced for the point cloud extraction. The first approach uses SfM orientation parameter on Agisoft Photo Scan while the second additionally takes into account building outlines digitized from the UAV data.

Our approach for orientation parameter calculation has four key steps. First, by using Agisoft Photo Scan we extracted a set of image orientation parameter $\left(\mathrm{X}_{0}, \mathrm{Y}_{0}, \mathrm{Z}_{0}\right.$, Omega, Phi, Kappa) of the UAV data in local coordinates system and calculate the differences with indirect georeferencing method (using GCP). Second, we calculated the image orientation parameters from building object as explained in section 2.3 , and calculated the difference with indirect georeferencing method (using GCP) as well. Third, we combine the image orientation parameters from building object to the indirect georeferencing method. Fourth, we compared the direct georeferencing method with the indirect georeferencing method in order to evaluate the usefulness of the building structure model for the final bundle adjustment.

\section{RESULTS AND DISCUSSIONS}

In this section, we describe our results namely the evaluation of the geometric accuracy and the orientation parameter accuracy. For our approach, two different image/photo processing software systems have been used. The first one is PCI Geomatics which concentrates on photogrammetric techniques for conventional aerial or satellite data processing. The other software is Agisoft Photo Scan which is relatively new to the market focusing on computer vision technology approaches.

The selected Ground Sampling Distance (GSD) for orthopotos is $5 \mathrm{~cm}$ and $10 \mathrm{~cm}$ for the DSM respectively. This resolution allows optimal zooming for manual interpretation and therefore high accuracy can be reached for providing $3 \mathrm{D}$ reference data in the final evaluation.

\subsection{Accuracy Assessments}

A DSM is a representation of the earth surface including manmade and natural structure above ground in three dimensional (3D) coordinates. The derived product of DSM which reflects the bare earth information is called Digital 
Terrain Model (DTM). In addition, the Ortho Rectified Imagery (ORI) can be produced as the ground projected object data by taking into account the DSM or DTM data.

With respect to the geometric accuracy, the National Standard for Spatial Data Accuracy (NSSDA) has been selected for geospatial positioning accuracy (FGDC, 1998). The main idea behind this method is the detection of blunders from a given data set and the derivation of a statistical model. In this case, Root Mean Square Errors (RMSE) can be used to estimate the absolute accuracy.

The RMSE can be calculated by the following equation (FGDC, 1998) for each corresponding object in the different datasets i.e. between the UAV data (Canon S-100) and the reference data (Leica RCD30). The calculation focuses on the point features, for the reason of simplicity with high certainty.

$$
\begin{aligned}
& \operatorname{RMSE}_{X}=\sqrt{\frac{\sum\left(X \mathrm{Re}_{i}-\text { XCheck }_{i}\right)^{2}}{n}} \\
& \operatorname{RMSE}_{Y}=\sqrt{\frac{\sum\left(Y \mathrm{Re}_{i}-\text { YCheck }_{i}\right)^{2}}{n}} \\
& \text { RMSE }_{r}=\sqrt{\text { RMSE }_{X}^{2}+R M S E_{Y}^{2}} \\
& \text { Accuracy }_{r}=1.7308 \times R M S E_{r} \\
& \text { RMSE }_{Z}=\sqrt{\frac{\sum\left(Z \mathrm{Re}_{i}-Z_{r} \text { Check }_{i}\right)^{2}}{n}} \\
& \text { Accuracy }_{Z}=1.96 \times R M S E_{Z}
\end{aligned}
$$

where:

$\operatorname{RMSE}_{\mathrm{x}}=$ Root Mean Square Error in $\mathrm{x}$ axis direction RMSE $_{\mathrm{y}}=$ Root Mean Square Error in y axis direction $\mathrm{RMSE}_{\mathrm{r}}=$ Horizontal (2D) Root Mean Square Error $\mathrm{RMSE}_{\mathrm{Z}}=$ Vertical (3D) Root Mean Square Error

$\left(\mathrm{XRe}_{\mathrm{i}}, \mathrm{YRe}_{\mathrm{i}}, \mathrm{ZRe}_{\mathrm{i}}\right)=$ Coordinates of check-points $\mathrm{i}$ in the reference dataset i.e. Leica RCD30 camera

$\left(\mathrm{XCheck}_{\mathrm{i}}\right.$, YCheck $_{\mathrm{i}}$, ZCheck $\left._{\mathrm{i}}\right)=$ Coordinates of check-points $\mathrm{i}$ in the UAV dataset (Canon S-100)

$\mathrm{n}=$ number of check-points

The accuracy is given at $95 \%$ confidence level. It means that $95 \%$ of the positions in the dataset will have an error with respect to true ground position that is equal to or smaller than the reported accuracy value.

For an appraisal, our DSM is compared directly with a similar DSM obtained by conventional airborne data acquisition using Leica RCD-30 metric camera. The comparison reveals that our approach can achieve decimeter level accuracy both in planimetric and vertical dimensions.

ICP Level 1 absolute accuracy assessment for the 2D (planimetric) and 3D (elevation) component has considered 10 checkpoints covering the test area provided from GNSS surveys as included in Table 3. For Direct Georeferencing (DG) from airborne acquisition, we did not use any GCP, while for either Indirect Georeferencing (IG) or combined method we use 3 GNSS monitoring stations and 5 post marking GNSS measurements as 8 GCPs. Since the DG method is not always free from the systematic errors such as GPS/INS-Camera misalignment, GPS time shift, camera calibration, etc, the

\begin{tabular}{|c|c|c|c|c|c|c|}
\hline \multirow[t]{2}{*}{ Camera } & \multicolumn{3}{|c|}{$\begin{array}{c}\text { Planimetric } \\
\text { Accuracy }(\mathrm{cm})\end{array}$} & \multicolumn{3}{|c|}{$\begin{array}{c}\text { Elevation } \\
\text { Accuracy }(\mathrm{cm})\end{array}$} \\
\hline & DG & IG & Co & DG & IG & $\mathrm{Co}$ \\
\hline RCD30 & 24.35 & 25.67 & 15.32 & 33.46 & 34.35 & 21.76 \\
\hline $\begin{array}{l}\text { Trimble } \\
\text { Phase } \\
\text { One }\end{array}$ & 58.43 & 47.35 & 41.32 & 59.36 & 56.35 & 42.46 \\
\hline $\begin{array}{c}\text { Canon S- } \\
100\end{array}$ & - & 44.35 & 37.35 & - & 51.35 & 40.25 \\
\hline
\end{tabular}
combined method using both GPS/INS data and GCPs is also applied. From ICP Level 1 assessment, we are convinced that our Leica RCD30 data can be used as a proper reference data for the UAV Canon S-100 data as well as for the Trimble Phase One data.

Table 3. ICP Level 1 (DG:Direct Georeferencing, IG:Indirect Georeferencing, Co:Combined DG-IG)

ICP Level 2 accuracy assessments have been done by using Leica RCD30 as the reference data. The generated Orthopoto and DEMs from airborne Trimble Phase One and UAV Canon S-100 were validated against Leica RCD30 data. To investigate the influence of the building structure in our approach the final assessment was performed in which the results from our approach were directly compared to the reference accuracy from direct georeferencing.
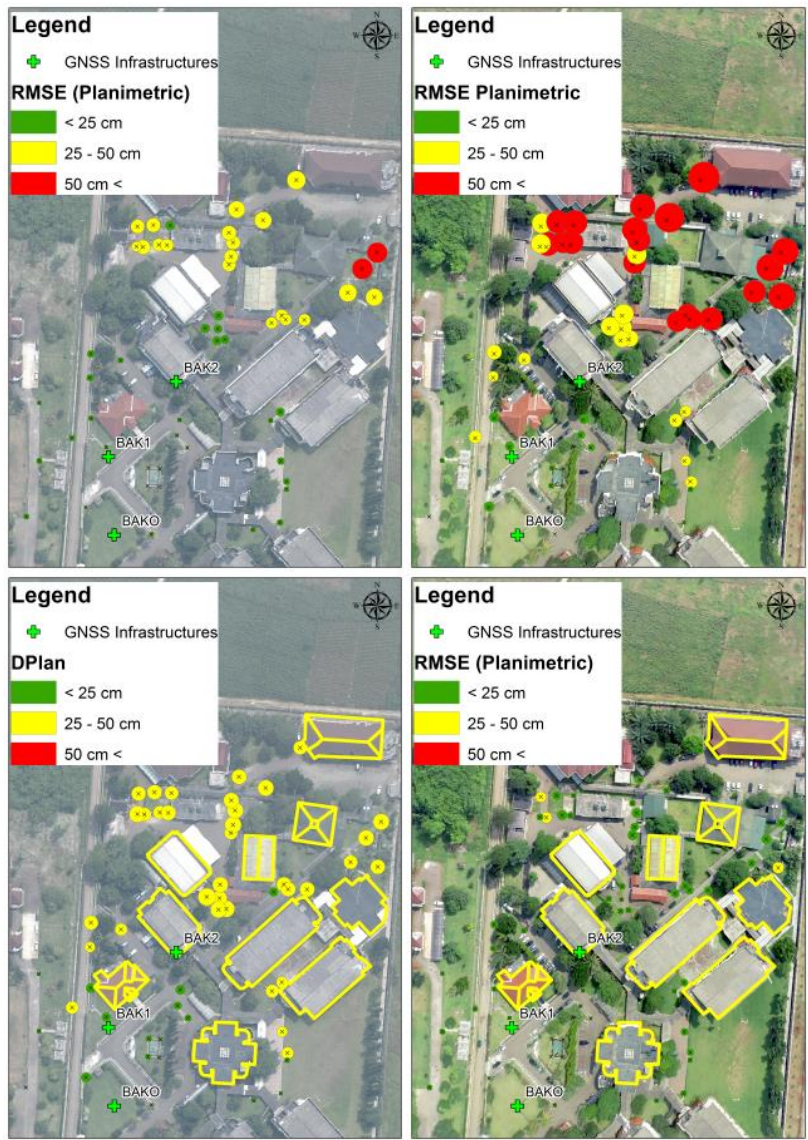

Figure 8. Accuracy Assessment: DG Trimble Phase One (upper-left), Combined Canon S-100 (upper-right), Combined (10 Buildings) Trimble Phase One (lower-left) and Combined (10 buildings) Canon S-100 (lower-right)

Combining the direct georeferencing (GPS/INS data) and indirect georefencing ( $8 \mathrm{GCPs}$ ) the empirical deviation from 52 
checkpoints manually selected from the orthophoto are in the range of $34 \mathrm{~cm}$ (planimetric) and $37 \mathrm{~cm}$ (elevation) respectively (Table 4). To justify the influence of building structures, all the 52 Checkpoints are selected outside the GCP perimeter (Figure 8). For instance, as also depicted in Figure 8, the effect of 10 building structures in the orientation parameter calculations using combined method for each Trimble Phase One and Canon S-100 has improved the RMSE (planimetric) in the range below $50 \mathrm{~cm}$. Obviously, the detail result of ICP Level 2 accuracy assessment in Table 4 shows us that there is a significant improvement if we are using building data structures as the additional reference to calculate external orientation parameters.

\begin{tabular}{|l|c|c|c|c|}
\hline \multirow{2}{*}{ Approach } & \multicolumn{2}{|c|}{ Trimble Phase One } & \multicolumn{2}{c|}{ Canon S-100 } \\
\cline { 2 - 5 } & $\begin{array}{c}\text { 2D } \\
(\mathrm{cm})\end{array}$ & $\begin{array}{c}\text { 3D } \\
(\mathrm{cm})\end{array}$ & $\begin{array}{c}2 \mathrm{D} \\
(\mathrm{cm})\end{array}$ & $\begin{array}{c}\text { 3D } \\
(\mathrm{cm})\end{array}$ \\
\hline DG & 55.34 & 64.54 & - & - \\
IG (3 GCP) & 76.53 & 85.28 & 65.53 & 75.13 \\
IG (8 GCP) & 68.35 & 69.24 & 47.43 & 49.23 \\
Co (1 Building) & 44.27 & 46.34 & 42.37 & 45.45 \\
Co (10 Buildings) & 35.37 & 38.34 & 34.32 & 37.64 \\
\hline
\end{tabular}

Table 4. ICP Level 2 (DG:Direct Georeferencing, IG:Indirect Georeferencing, Co:Combined DG-IG)

\subsection{Orientation Parameter Assessments}

As already explained in section 2.3, image external orientation parameter extraction in this paper uses the geometrical consideration from local building structure interactively. Structure from Motion (SfM) is helpful to provide the initial building structure.

Obviously, geometrical accuracy of the building structure is the pre-requisite for performing $3 \mathrm{D}$ analysis on high resolution data. Initially, we put a posteriori external orientation parameters given by the GPS/INS equipment for each camera in the Table 5 .

\begin{tabular}{|c|c|c|c|}
\hline $\begin{array}{c}\text { Orientation } \\
\text { Parameter }\end{array}$ & RCD30 & $\begin{array}{c}\text { Trimble } \\
\text { Phase One }\end{array}$ & Canon S-100 \\
\hline $\mathrm{dX}_{0}(\mathrm{~m})$ & 0.008 & 0.135 & 33.843 \\
\hline $\mathrm{dY}_{0}(\mathrm{~m})$ & 0.011 & 0.140 & 10.069 \\
\hline $\mathrm{dZ}_{0}(\mathrm{~m})$ & 0.017 & 0.062 & 10.532 \\
\hline $\mathrm{dO}\left(^{\circ}\right)$ & 0.003 & 0.009 & - \\
\hline $\mathrm{dP}\left(^{\circ}\right)$ & 0.003 & 0.009 & - \\
\hline $\mathrm{dK}\left(^{\circ}\right)$ & 0.009 & 0.002 & - \\
\hline
\end{tabular}

Table 5. A posteriori external orientation parameters (Direct Georeferencing)

To evaluate the accuracy of orientation parameters, we used two overlapping images for airborne acquisition (Trimble Phase One/Leica RCD30) and four overlapping images for UAV acquisition (Canon S-100). From the comparison with Table 6, our approach can significantly improve the direct georeferencing accuracy for our UAV data (Canon S-100). In specific, our direct georeferencing approach can provide submeter for sensor position accuracy and maximum 0.309 degree for sensor orientation accuracy.

\begin{tabular}{|c|r|r|r|}
\hline $\begin{array}{c}\text { Orientation } \\
\text { Parameter }\end{array}$ & RCD30 & $\begin{array}{c}\text { Trimble Phase } \\
\text { One }\end{array}$ & Canon S-100 \\
\hline $\mathrm{dX}_{0}(\mathrm{~m})$ & 0.033 & 6.808 & 1.122 \\
\hline $\mathrm{dY}_{0}(\mathrm{~m})$ & 0.011 & 1.885 & 0.272 \\
\hline $\mathrm{dZ}_{0}(\mathrm{~m})$ & 0.030 & 1.506 & 2.893 \\
\hline $\mathrm{dO}\left(^{\circ}\right)$ & 0.009 & 0.183 & 0.237 \\
\hline $\mathrm{dP}\left(^{\circ}\right)$ & 0.041 & 0.362 & 0.309 \\
\hline $\mathrm{dK}\left(^{\circ}\right)$ & 0.022 & 0.012 & 0.137 \\
\hline
\end{tabular}

Table 6. External orientation parameters (Combined with Building structures calculation)

The refinement of external orientation parameters has improved the quality of resulted DEM as presented in Figure 9. The level of details of our UAV data in the final result is compatible with the Leica RCD30 medium-format camera.

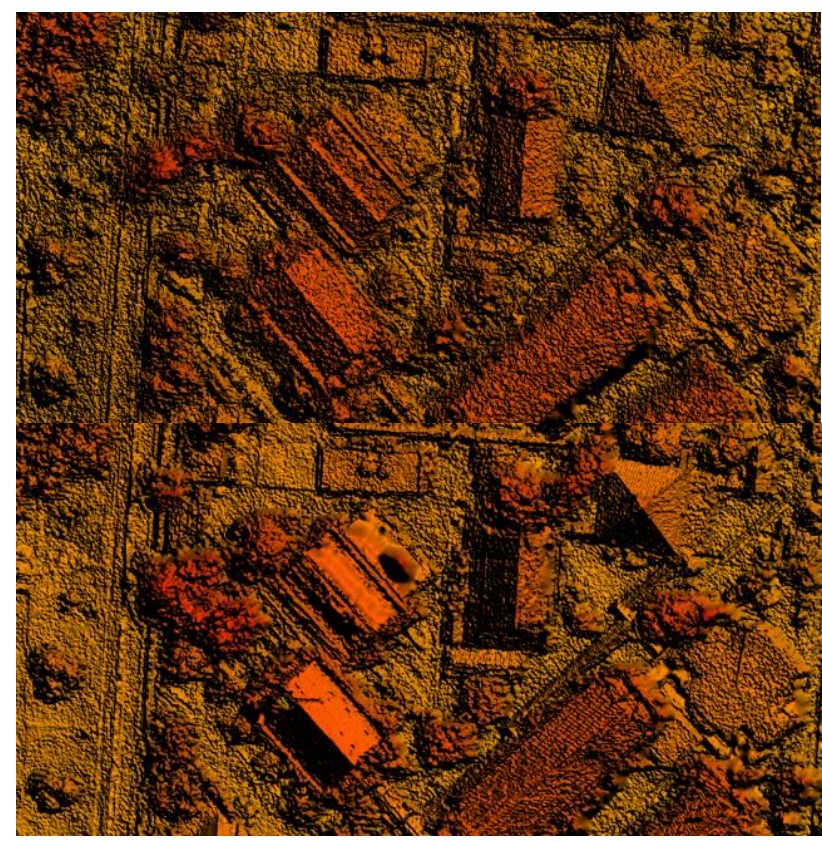

Figure 9. DSM in $10 \mathrm{~cm}$ GSD of BIG's office: UAV Canon S100 (upper) and Airborne RCD30 (lower)

\section{CONCLUSIONS}

In this paper, an alternative solution method to determine the camera exterior orientation parameters has been presented. We can notice the actual tendency in photogrammetry toward Structure from Motion (SfM). Proven that the determination of camera intrinsic and exterior parameters constitute the first step of the modelling procedure, the solutions based on projective geometry aspects can be very useful in this context. The recovered camera exterior parameters, using the building structure as a reference model, can improve the accuracy of combined DG/IG method for large scale topographical mapping applications. By only use three GCPs, our approach shows a better extrapolation for the area outside the GCP coverage once the building structure model has been taken into account in the orientation parameter calculation. Hence, topographic maps with absolute NSSDA (95\%) of decimetre (below $50 \mathrm{~cm}$ for planimetric and elevation aspects) can be produced by combined DG/IG method of UAV data with our simple building structure approach. This low cost approach can generate orthophotos and DSM sufficient enough for 1:5,000 Large 
Scale Topographical Mapping requirements in Indonesia. Other potential solutions using ground control features from existing databases such as orthophotos, DTM or 3D wireframe models of more complex man-made objects can be investigated in the future to enhance the results. The proposed method can be simply applied to strip and block adjustment as well.

\section{ACKNOWLEDGEMENTS}

We would like to acknowledge Geospatial Information Agency of Indonesia (BIG) for providing the Airborne Photogrammetric dataset (Leica RCD30, Trimble P65+) as well as the access to the GPS reference station (CORS) during the campaign over the BIG's office in Cibinong, Indonesia and necessary processing software licenses (PCI Geomatics, Agisoft Photo Scan).

\section{REFERENCES}

Agisoft, 2013. Agisoft PhotoScan User Manual: Professional Edition.Version1.0.0.

http://downloads.agisoft.ru/pdf/photoscan-pro_1_0_0_en.pdf

(accessed 14 Apr. 2016)

Cramer, M, Stallmann, D., Haala, N., 2000. Direct Georeferencing Using GPS/Inertial Exterior Orientations for Photogrammetric Applications. International Archives of Photogrammetry and Remote Sensing, Amsterdam, The Netherlands, Vol. 33, Part B3, pp. 198-205.

FGDC, 1998. Geospatial Positioning Accuracy Standards Part 3: National Standard for Spatial Data Accuracy (version 2.0), Washington, D.C., FGDC-STD-001-1998: Federal Geographic Data Committee, pp.3-4.

Höchle, J., 2012. Medium-Format Cameras and Their Use in Topographic Mapping. The International Annals of the Photogrammetry, Remote Sensing and Spatial Information Sciences. Vol. I-4, pp.77-82.

Javernick, L., Brasington, J., Caruso, B., 2014. Modeling the topography of shallow braided rivers using Structure-fromMotion Photogrammetry. Geomorphology 213. pp. 166-282.

Westoby, M.J., Brasington, J., Glasser, N.F., Hambrey, M.J., Reynolds, J.M., 2012. Structure-from-Motion photogrammetry: a low-cost, effective tool for geoscience applications. Geomorphology 179, pp.300-314.

Tampubolon, W., and Reinhardt, W., 2014. UAV Data Processing for Large Scale Topographical Mapping. Int. Arch. Photogramm. Remote Sens. Spatial Inf. Sci., XL-5, pp. 565572 .

Zhang, Z., Wu, J., Zhang, Y., Zhang, Y. And Zhang, J., 2004. Multi-View 3D City Model Generation with Image Sequences. International Archives of Photogrammetry and Remote Sensing, Istanbul, Turkey, Vol. 34, Part 5, pp. 351-356. 